\title{
Arctic-Mid-Latitude Linkages in a Nonlinear Quasi-Geostrophic Atmospheric Model
}

\author{
Dörthe Handorf, ${ }^{1}$ Klaus Dethloff, ${ }^{1}$ Sabine Erxleben, ${ }^{1}$ \\ Ralf Jaiser, ${ }^{1}$ and Michael V. Kurgansky ${ }^{2}$ \\ ${ }^{1}$ Alfred Wegener Institute for Polar and Marine Research, Research Unit Potsdam, Telegrafenberg A43, \\ 14473 Potsdam, Germany \\ ${ }^{2}$ A.M. Obukhov Institute of Atmospheric Physics, Russian Academy of Sciences, Pyzhevsky 3, \\ Moscow 119017, Russia
}

Correspondence should be addressed to Dörthe Handorf; doerthe.handorf@awi.de

Received 20 January 2017; Revised 10 May 2017; Accepted 23 May 2017; Published 13 June 2017

Academic Editor: Yoshihiro Tomikawa

Copyright @ 2017 Dörthe Handorf et al. This is an open access article distributed under the Creative Commons Attribution License, which permits unrestricted use, distribution, and reproduction in any medium, provided the original work is properly cited.

\begin{abstract}
A quasi-geostrophic three-level T63 model of the wintertime atmospheric circulation of the Northern Hemisphere has been applied to investigate the impact of Arctic amplification (increase in surface air temperatures and loss of Arctic sea ice during the last 15 years) on the mid-latitude large-scale atmospheric circulation. The model demonstrates a mid-latitude response to an Arctic diabatic heating anomaly. A clear shift towards a negative phase of the Arctic Oscillation (AO-) during low sea-ice-cover conditions occurs, connected with weakening of mid-latitude westerlies over the Atlantic and colder winters over Northern Eurasia. Compared to reanalysis data, there is no clear model response with respect to the Pacific Ocean and North America.
\end{abstract}

\section{Introduction}

In the Northern Hemisphere $(\mathrm{NH})$, recent climate change is most pronounced in the Arctic. Here, an unprecedented decrease in the sea-ice-cover (e.g., [1]) and a pronounced air temperature rise are observed during the last 15 years (e.g., [2]), which constitutes the essence of Arctic amplification (AA) (e.g., [3, 4]). Although the causes of AA are not well understood, especially with regard to the relative role of oceanic circulation, it remains a withstanding problem to explain a link between AA and anomalous mid-latitude wintertime circulation patterns. These linkages manifest themselves as a sequence of unusually cold winters observed during the last years over Northern Eurasia and North America $[5,6]$. There is a growing body of works relating anomalously low summer-autumn sea-ice-cover in the Arctic to severe winters over Northern Eurasia and North America based on both reanalysis data and model results analysis (e.g., [7, 8]; review articles by Cohen et al. [9-11]). Most evidence has been found for dynamical pathways in the troposphere and in the stratosphere, resulting in more frequent occurrence of the negative phase of the Arctic Oscillation (AO) or North Atlantic Oscillation (NAO) in late winter (e.g., $[5,12,13])$. Due to additional heat uptake by the larger sea-ice-free areas in summer and early autumn, the refreezing in autumn is delayed. This leads to additional ocean heat release, especially to the Arctic atmospheric boundary layer in autumn. Therefore, the vertical stability decreases, which may lead to amplified baroclinic systems over the Arctic [12]. The anomalous transient eddy forcing by the amplified baroclinic systems may force planetary waves [12] and may affect the onset and maintenance of the Siberian High. Additionally, the Siberian High may be strengthened in autumn by increased Siberian snow cover due to oceanic moisture release to the Arctic atmosphere $[14,15]$.

In late autumn, the increased Siberian snow cover and the persistent sea-ice anomalies in particular over the Barents/Kara Seas provide diabatic heating sources resulting in direct forcing of and constructive interference with existing tropospheric planetary Rossby waves in this month $[7,14]$. On the background of a reduced meridional temperature gradient, these conditions favour a more frequent occurrence 
of meridional flow regimes like negative phase of $\mathrm{AO}$ in early and mid-winter which may persist into late winter. The stronger tropospheric planetary waves induce stronger vertical propagation of wave energy and initiate a stratospheric pathway [14]. Enhanced upward propagating planetary waves may lead to wave breaking in the polar stratosphere, which in turn may weaken the polar vortex (e.g., $[16,17])$. The downward propagation of these stratospheric circulation anomalies may start in mid-winter and can contribute to the persistent negative $\mathrm{NAO} / \mathrm{AO}$ anomalies in mid-winter to late winter $[13,16,18]$.

However, the effect of AA on mid-latitude circulation systems in reanalysis and comprehensive GCMs is entangled by the influence of additional factors such as oceanatmosphere interactions and tropical atmospheric forcing and the internal variability of the atmosphere and the coupled system itself. These issues complicate investigation of the dynamical mechanism of Arctic-mid-latitude linkage and suggest an approach using a hierarchy of models ranging from highly simplified to comprehensive GCMs together with observations. In such a hierarchical approach, some of these additional influencing factors and mechanisms can be bypassed. Hassanzadeh et al. [19] have recently emphasized this point and applied an idealized dry GCM with the physical parameterizations and forcings based on Held and Suarez [20] to study the impact of AA on mid-latitude blocking and waviness. Following a similar approach, we use here an idealized nonlinear quasi-geostrophic (QG) hemispheric model of atmospheric circulation with spectral resolution T63 and three vertical levels (QGT63L3). This model has been proven to be efficient in reproducing atmospheric circulation regimes in the NH [21]. This is applicable to study Arctic-midlatitude linkages, since they manifest as large-scale circulation changes as discussed before. Therefore, a coarse vertical resolution with three levels may be conjectured as adequate for capturing the main features of these linkages. The issue of the model's spatial resolution was addressed in Labsch et al. [21]. In particular, it was emphasized by Labsch et al. [21] that we approach the limit of applicability of the quasi-geostrophic approximation by increasing the spectral resolution in the model to T63, because the Rossby number on the smallest resolved scale becomes comparable to unity. From this perspective, keeping a relatively coarse vertical resolution is beneficial, because it permits the decoupling of horizontal motion on the smallest resolved scales between different vertical levels, when the thermal wind equation, which is responsible for this coupling, ceases to be valid. In effect, the smaller-scale motions obey barotropic dynamics at each model level and, as such, we have a seamless transition of quasi-geostrophic dynamics operating at larger baroclinic scales, which are satisfactorily described in our three-level model, to barotropic dynamics acting on the smaller scales.

The objective of our study is to investigate the impact of AA on the mid-latitude circulation with a simple but dynamically consistent atmospheric GCM. We intend to demonstrate the QGT63L3 capability of reproducing changes in midlatitude circulation features that accompany AA. This demonstration will serve an additional proof for the reproducibility of these changes in a hierarchy of GCMs and will reveal an essentially quasi-geostrophic dynamics underlying AA-midlatitude circulation linkage. As a result, our study will provide insights into the role of quasi-geostrophic dynamics for AAmid-latitude circulation linkages, which otherwise remains obscure when using comprehensive GCMs.

\section{Model Description}

The three-level model [21-23] simulates the QG evolution of $\mathrm{NH}$ atmospheric flow stream functions at the three vertical levels, $167 \mathrm{hPa}, 500 \mathrm{hPa}$, and $833 \mathrm{hPa}$, under perpetual winter (December-January-February, DJF) conditions. The lower two levels represent the lower and middle troposphere, while the upper level mimics the upper troposphere and the lower stratosphere. It is a hemispheric model with a T63 horizontal spectral resolution $\left(\sim 1.875^{\circ} \times 1.875^{\circ}\right)$. Northern Hemisphere's topography adapted to T63 resolution (Figure 1 in [21]) acts as orographic forcing. Diabatic heating is established by a thermal relaxation, with a timescale of 22.7 days, towards predefined radiative equilibrium temperature fields at the model pressure levels of 333 and $667 \mathrm{hPa}$. An additional surface friction mechanism damps the $833 \mathrm{hPa}$ stream function towards a predefined zonally symmetric surface forcing function [24] with a timescale of 1.08 days. A horizontal scale-selective $\nabla^{6}$ hyperdiffusion is used with the shortest e-folding time of $48.5 \mathrm{~h}$ ( $\approx 2$ days) for the maximal total wavenumber $N=63$. The vertical temperature lapse rate has been fixed to $3.0 \mathrm{~K} / \mathrm{km}$ at $333 \mathrm{hPa}$ and $6.5 \mathrm{~K} / \mathrm{km}$ at $667 \mathrm{hPa}$.

Both nonzonal and zonal components of the radiative equilibrium temperature fields (the latter together with the surface forcing) have been tuned to generate realistic patterns of nonzonal extratropical diabatic heating and zonal wind fields for mean climatological winter conditions. After tuning, the diabatic heating field is kept constant over time.

The target nonzonal parts of wintertime (DJF) diabatic heating rates over the $\mathrm{NH}$ are derived from ERA-Interim reanalysis for high (1979-2000) and low (2001-2014) Arctic sea-ice conditions at $300 \mathrm{hPa}$ and $700 \mathrm{hPa}$ which are the closest to the model pressure levels pf $333 \mathrm{hPa}$ and $667 \mathrm{hPa}$ and are shown in Figure 1. The atmospheric ERA-Interim reanalysis data have been provided by the European Centre for Medium-Range Weather Forecasts ([25]; http://apps.ecmwf .int/datasets/). The nonzonal parts of diabatic heating rates have been attenuated near the equator (Figure 1). Diabatic heating is determined by calculating the thermodynamic equation in each layer, which involves temperature tendency and three-dimensional temperature advection. Figure 1 also displays the difference between these two diabatic heating patterns, indicating regionally varying cooling and warming structures. After tuning, the diabatic heating fields are still very similar to the target fields derived from ERAInterim reanalysis (not shown). The target zonally averaged zonal wind climatology for the above-indicated time periods derived from ERA-Interim is presented in Figure 2. Other details of the tuning procedure for QGT63L3 are given by Labsch et al. [21]. 


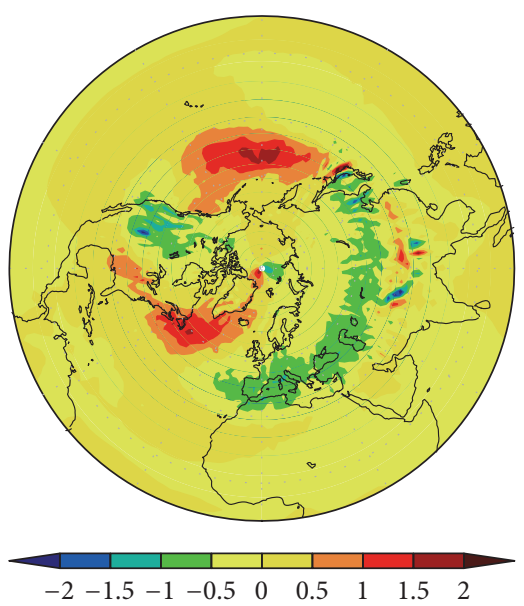

(a)

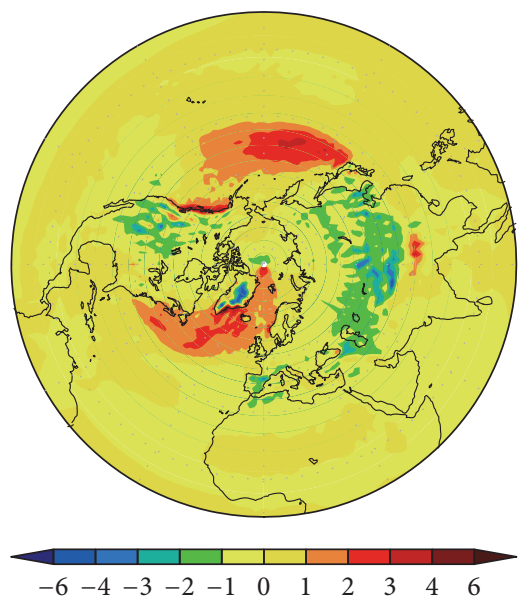

(d)

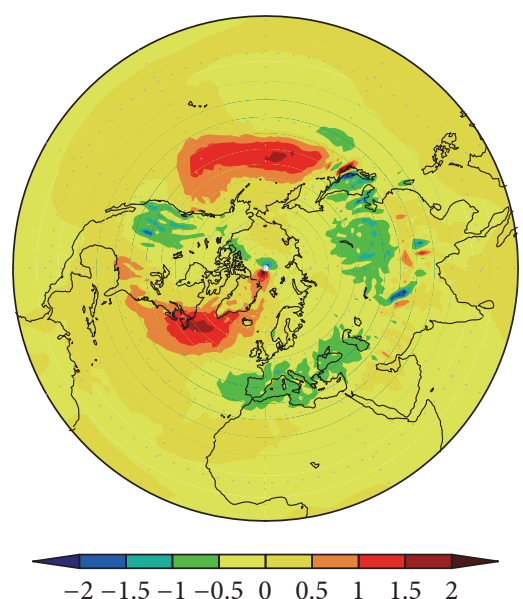

(b)

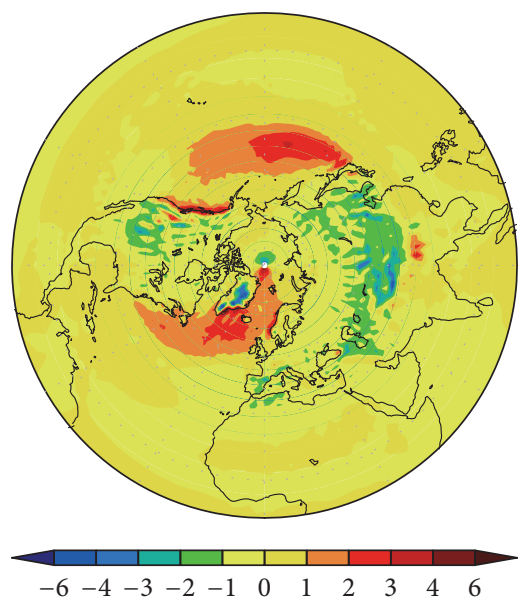

(e)

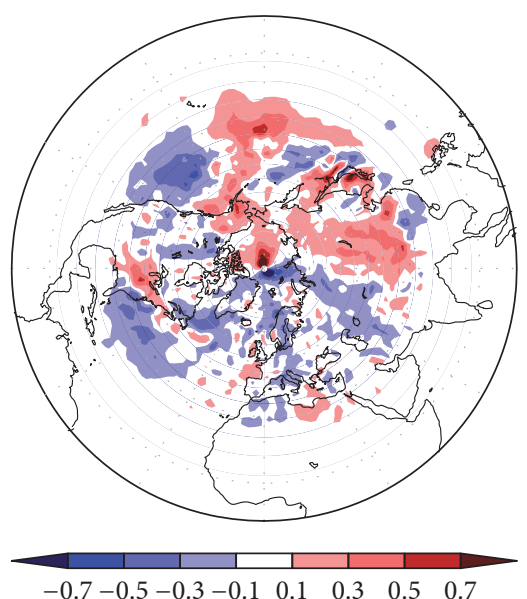

(c)

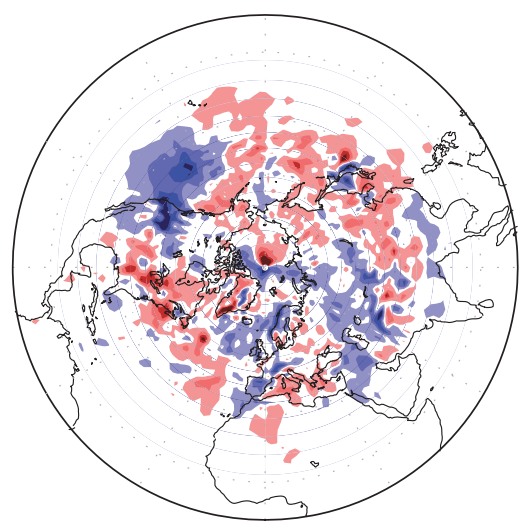

$\begin{array}{llllll}-0.7 & -0.5-0.3-0.1 & 0.1 & 0.3 & 0.5 & 0.7\end{array}$

(f)

FIGURE 1: Wintertime (DJF) extratropical nonzonal diabatic heating $\left(\mathrm{W} / \mathrm{m}^{2}\right)$ at ((a), (b), and (c)) 300 and ((d), (e), and (f)) $700 \mathrm{hPa}$ derived from ERA-Interim reanalysis. At each row, the figures correspond to the low ((a) and (d)) and high ((b) and (e)) Arctic sea-ice-cover conditions and ((c) and (f)) to their difference (low minus high).

It has to be emphasized that the used target diabatic heating fields accumulate all forcings, which act in the atmosphere, not only due to the Arctic sea-ice loss. By attenuating the diabatic heating field near the equator, we smooth out the direct influence of the tropical forcing, although its remote influence remains in the model.

Using the forcings described above, two 20,000-day model integrations were performed for the high and low Arctic sea-ice conditions, respectively. The last 18,000 days ( $\sim 50$ years) of integration were used to evaluate the model climatology and to perform analysis to investigate the impact of AA.

\section{Methods}

In order to evaluate the model climatology, we compare the model results with the atmospheric ERA-Interim reanalysis data [25]. ERA-Interim data have been calculated with spectral resolution of T255 on 60 vertical levels, with the model top at $0.1 \mathrm{hPa}$. Here, we used the geopotential height fields interpolated to 37 pressure levels up to $1 \mathrm{hPa}$ on a $2^{\circ} \times 2^{\circ}$ latitude/longitude grid from 1979 to 2014. The geopotential height fields which correspond to the three layers of the QG model have been calculated by mass-weighted averaging over three atmospheric layers, where each layer covers approximately one-third of the total atmospheric mass.

To characterize the changes in planetary waves, we have calculated the zonal wavenumber-1 component of the mean $\mathrm{NH}$ geopotential height fields at each level. To obtain the wavenumber-1 component, we have performed spherical harmonic analysis to the full geopotential height fields and spherical harmonic synthesis with truncation at total wavenumbers-1 at each level afterwards. These calculations have been carried out by using subroutines from the software package SPHEREPACK 3.2 [26].

For the determination of the most dominant atmospheric variability pattern, namely, the $\mathrm{AO}$, an Empirical Orthogonal Function (EOF) analysis has been applied (e.g., [27]; see also 


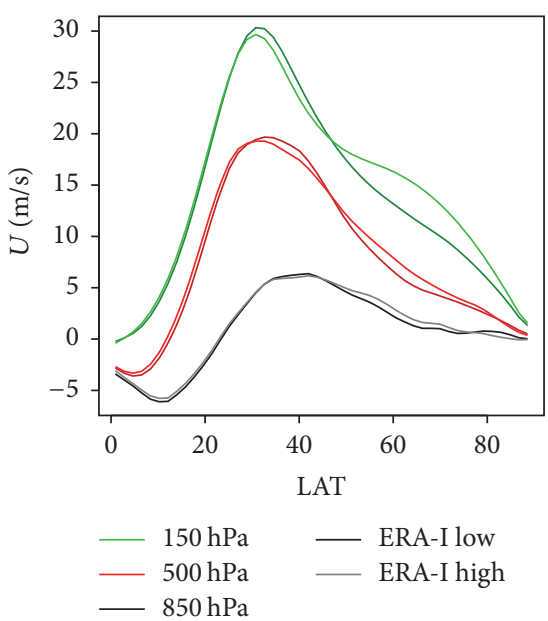

(a)

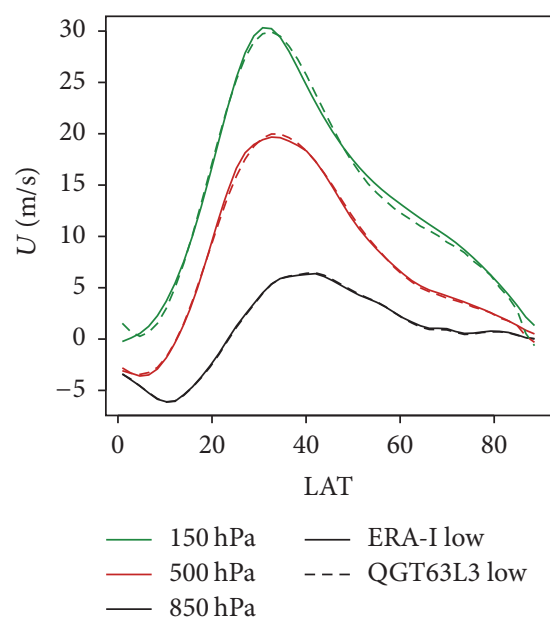

(b)

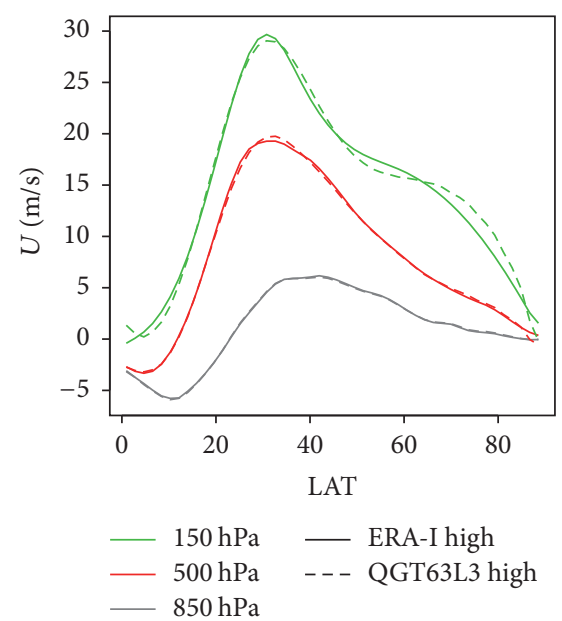

(c)

Figure 2: (a) The target (observed) time-mean zonal wind profiles $(\mathrm{m} / \mathrm{s})$ for the three model levels. The dark and light lines correspond to low and high Arctic sea-ice-cover conditions, respectively; the green, red, and black/grey color of lines refers to the upper, middle, and lower levels, correspondingly. (b) The target (observed, solid lines) and simulated (dashed lines) time-mean zonal wind profiles ( $\mathrm{m} / \mathrm{s}$ ) for the three model levels for low Arctic sea-ice-cover conditions. (c) The same as in (b) but for high Arctic sea-ice-cover conditions.

[28]). The corresponding time series (or principal components, PCs) have been obtained by projecting the geopotential height fields onto the EOF patterns and describe the temporal behavior of the atmospheric variability patterns. Equal-area weighting is ensured by multiplying the fields with the square root of the cosine of latitude before calculating the EOFs. EOF patterns and PC time series are renormalized such that the corresponding PC time series are standardized (cf. [29]).

Here, the first EOF has been calculated for each atmospheric layer by combining both single 50-year simulations for low and high sea-ice conditions. The corresponding three PC time series have been analyzed with the aim to detect shifts in the frequency of occurrence of positive or negative phases of the most dominant variability pattern. This was done by searching for changes in the mean PC values as well as in the histogram of the corresponding PC time series.

\section{Model Results}

To evaluate the model climatology, Figures 2(b) and 2(c) display the time-mean zonal wind profiles of the model simulations for the low and high sea-ice conditions, respectively, together with zonal winds taken from the ERA-Interim data for the winter (DJF) months. The agreement between the modeled and observed profiles at the lower and middle level is very good for both high and low sea-ice conditions (root mean square errors less than $0.08 \mathrm{~m} / \mathrm{s}$ at lower level and less than $0.28 \mathrm{~m} / \mathrm{s}$ at middle level). At the upper level, the modeled wind profiles show larger deviation near the equator and at high latitudes in particular for the high ice simulations. Anyway, the wind profiles are reproduced satisfactorily with root mean square errors less than $0.97 \mathrm{~m} / \mathrm{s}$.

The evaluation of geopotential height fields is presented in Figure 3. Figures 3(a), 3(b), and 3(c) show the mean geopotential height at $833 \mathrm{hPa}$ surface, the lower model level for the indicated model runs, and also the difference (low minus high sea-ice-cover) between the geopotential height fields. Figures 3(d), 3(e), and 3(f) display the corresponding massweighted geopotential height fields taken from ERA-Interim reanalysis and averaged over the lowest vertical layer that covers approximately one-third of the total atmospheric mass.

Figure 4 shows the same patterns as in Figure 3 but at the $167 \mathrm{hPa}$ level together with mass-averaged reanalysis data corresponding to the upper vertical layer of the atmosphere. The patterns of mean geopotential height of the middle model level at $500 \mathrm{hPa}$ and the corresponding geopotential height fields taken from ERA-Interim reanalysis resemble those in Figure 3 and are not shown here.

As visible in Figure 3, the model quite satisfactorily reproduces the mean climatology in the troposphere, including the wave- 2 circumpolar positive anomaly structure at the lower level, orientated from Greenland to Siberia. It is also performing well as far as it regards a westward shift of atmospheric circulation patterns for low sea-ice-cover conditions reported by Handorf et al. [30], as seen in the structure of the difference of wave-1 component of the geopotential height at $833 \mathrm{hPa}$ (Figure 5). Figure 3 qualitatively shows that the model correctly reproduces a tendency towards the negative phase of the Arctic Oscillation ( $\mathrm{AO}-$ ) during low-ice conditions. This implies weakening of mid-latitude westerlies over Atlantic, an enhancement of Siberian High (cf. [31]), and presumably more cold winters over the Northern Eurasia. A closer inspection of Figure 4 confirms this tendency, since the positive geopotential height anomaly (low minus high seaice-cover) around the pole at the $167 \mathrm{hPa}$ level in Figure 4 describes the weakening of the stratospheric polar vortex and corresponds to a negative Arctic Oscillation-like pattern in the troposphere (cf. [32]). The results concerning Pacific Ocean and North America do not show a clear signal. 

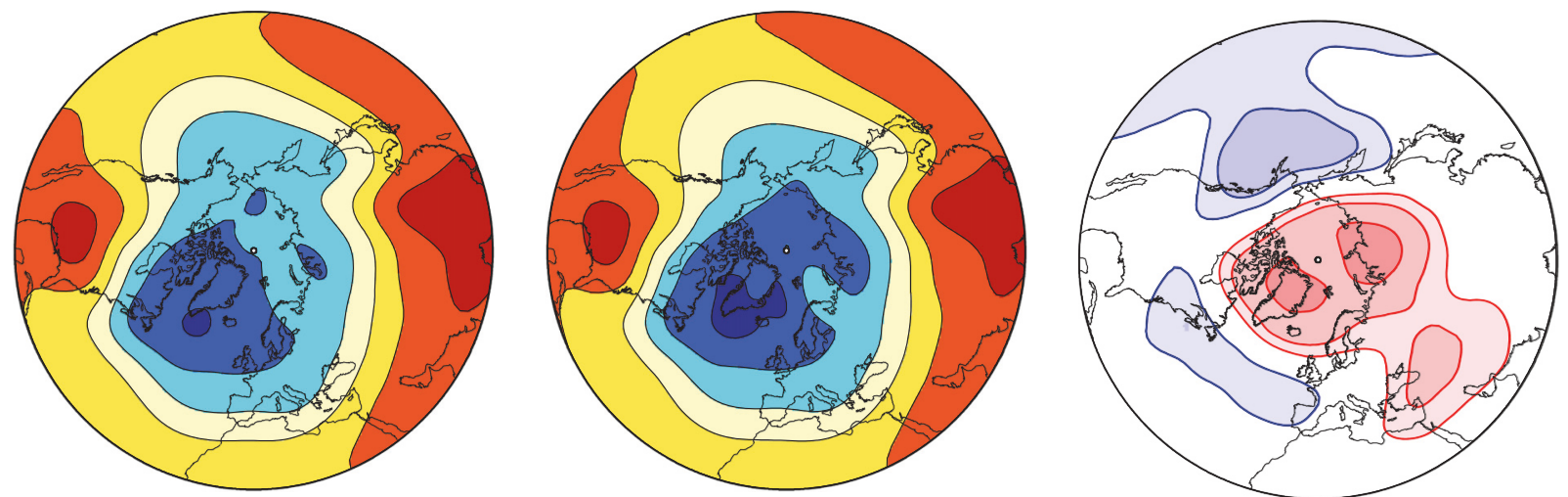

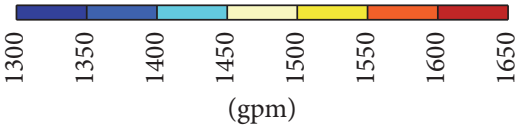

(a)
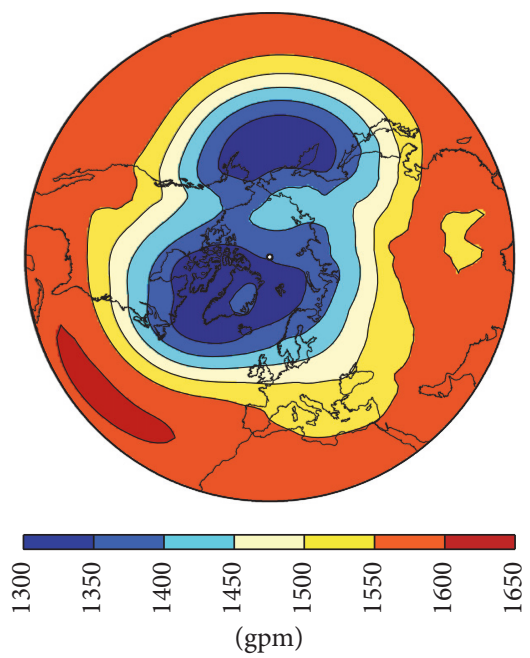

(d)

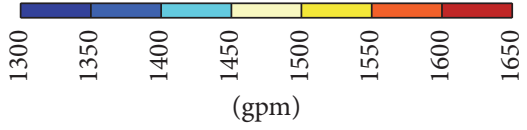

(b)

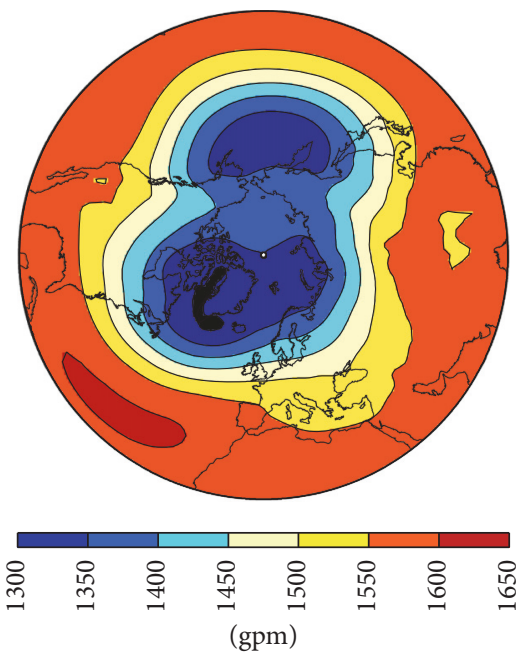

(e)

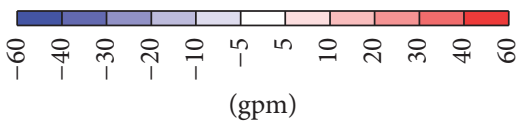

(c)
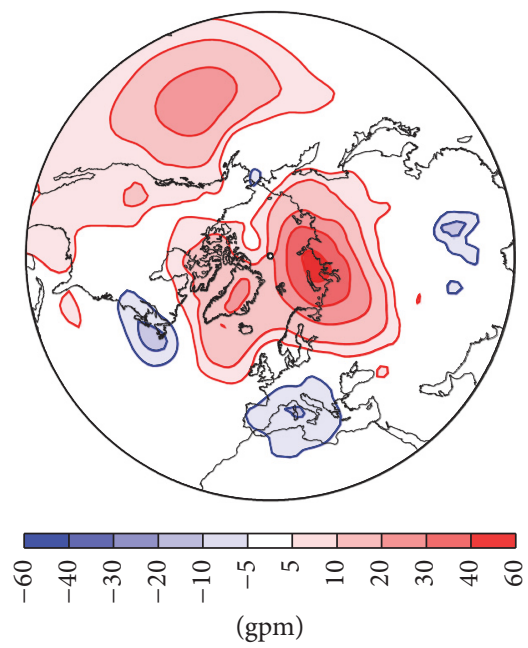

(f)

Figure 3: Mean geopotential height (gpm) of the $833 \mathrm{hPa}$ model level ((a), (b), and (c)) and from the layer-averaged ERA-Interim reanalysis data ((d), (e), and (f)). At each row, the figures correspond to the low ((a) and (d)) and high ((b) and (e)) Arctic sea-ice-cover conditions and ((c) and (f)) to their difference (low minus high).

Figure 6 displays the first EOF at the three model levels, which was computed by combining both single 50 -year simulations for low and high sea-ice conditions. A clear barotropic structure resembling the $\mathrm{AO}$ is visible. The corresponding three PC time series shown in Figure 7 indicate a clear shift from more frequent occurrences of the positive $\mathrm{AO}$ phase in the first time slice to the more frequent occurrence of a negative AO phase in the second time slice. This result is underpinned by the histogram of the corresponding PC time series at the model levels in Figure 8.

\section{Concluding Remarks}

By using a nonlinear quasi-geostrophic spectral model of wintertime atmospheric circulation over the Northern Hemisphere, it is shown that Arctic amplification is connected with distinct and clearly interpretable mid-latitude circulation changes over the Atlantic Ocean and Northern Eurasia. In full accord with ERA-Interim reanalysis data, a tendency towards a negative phase of Arctic Oscillation (AO-) during low sea-ice-cover conditions has been revealed, with weakening of mid-latitude westerlies over Atlantic and, consequently, colder winters over Europe and Northern Eurasia. At the upper level, which mimics the lower stratosphere, the modeling results show the polar vortex weakening that accompanies AA, which is consistent with negative phases of Arctic Oscillation and North Atlantic Oscillation (Figure 4).

Our modeling results significantly disagree with reanalysis data over the Pacific Ocean and west coast of Northern America, because a negative geopotential height response to AA is modeled in the troposphere contrary to the positive geopotential height response in reanalysis (Figure 3). On the other hand, our results for the Pacific are in general agreement (although details of the negative anomaly spatial distribution 

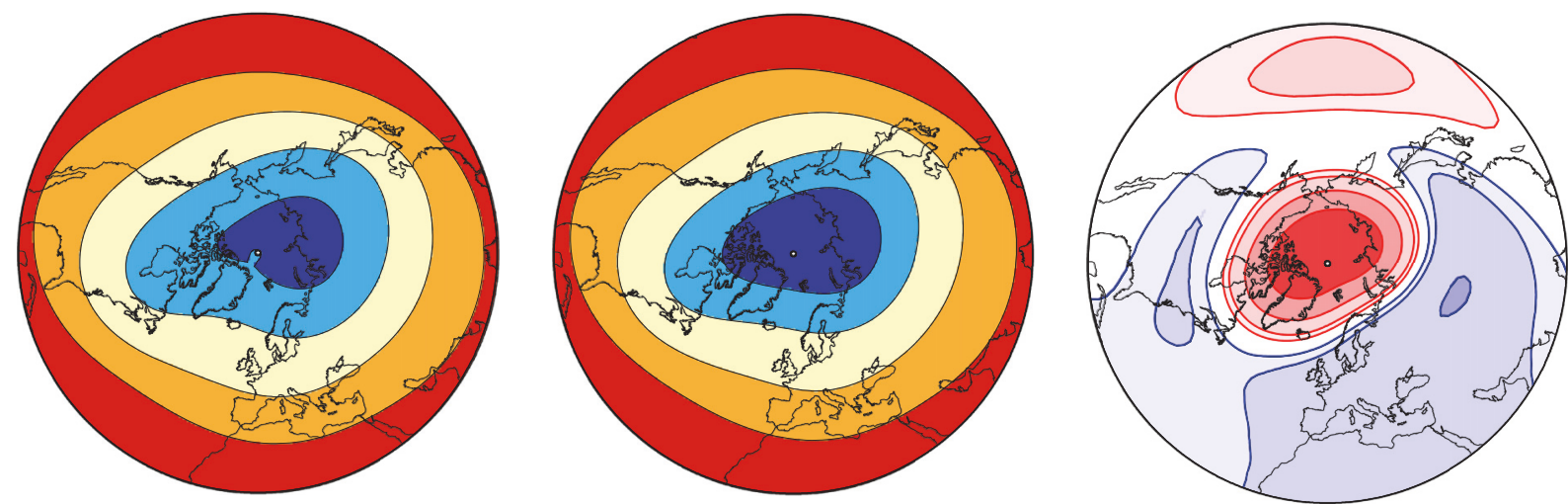

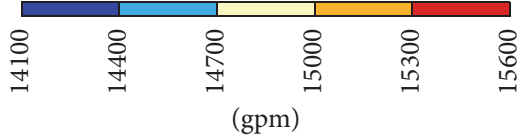

(a)
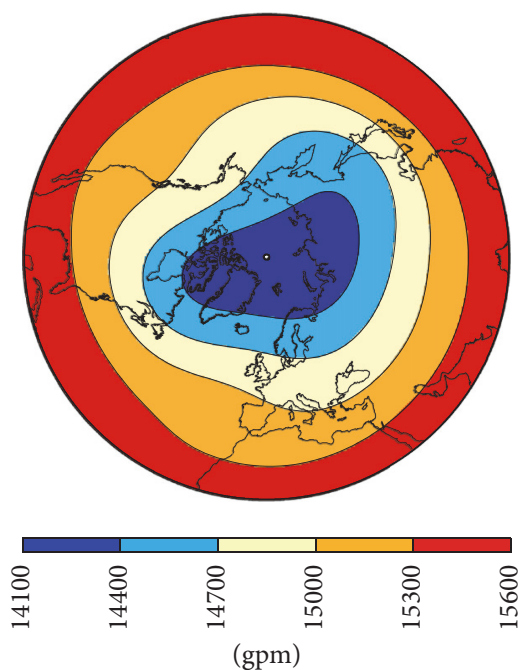

(d)

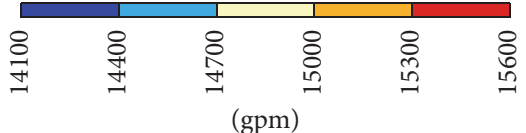

(b)
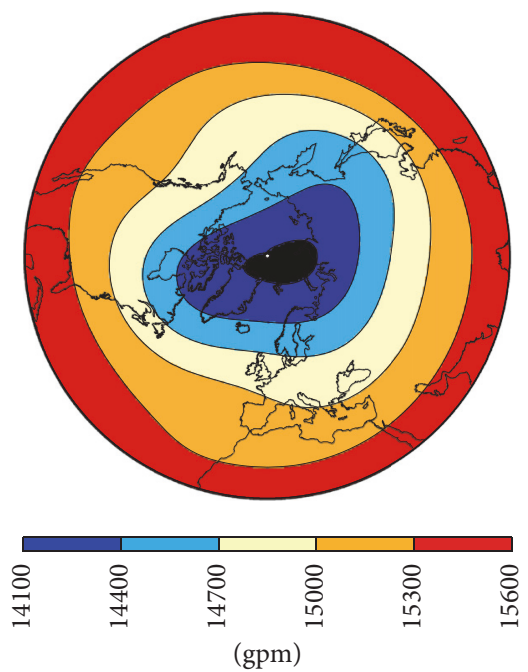

(e)

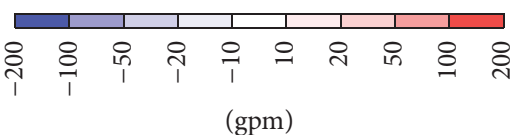

(c)
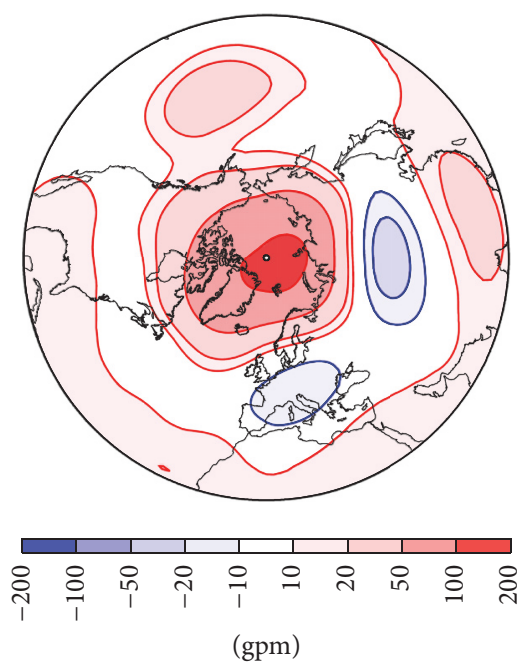

(f)

FIGURE 4: The same as in Figure 3 but for the $167 \mathrm{hPa}$ model level.

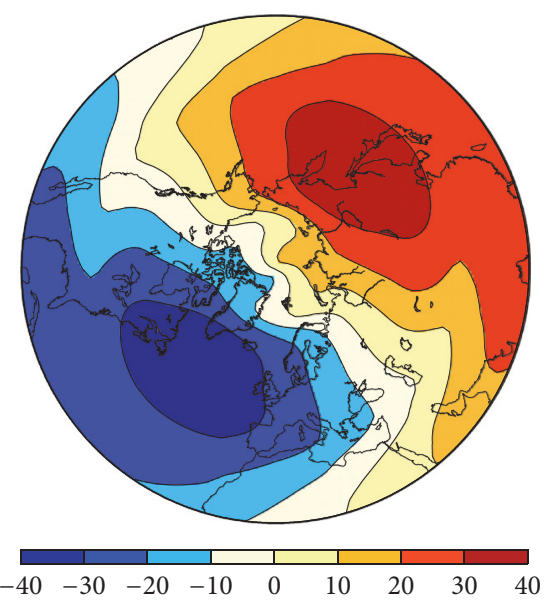

(a)

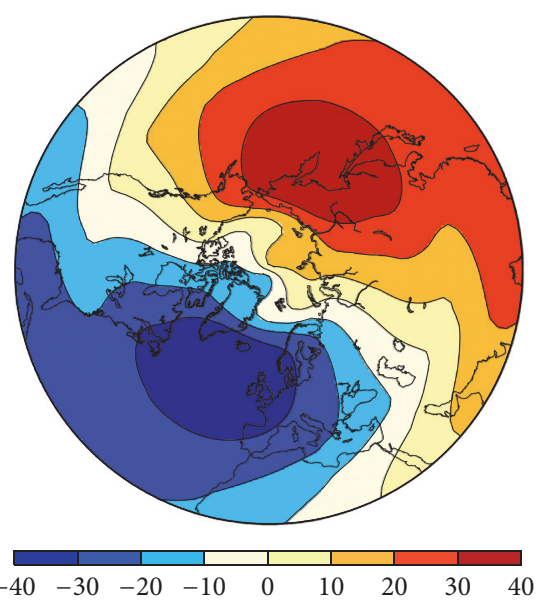

(b)

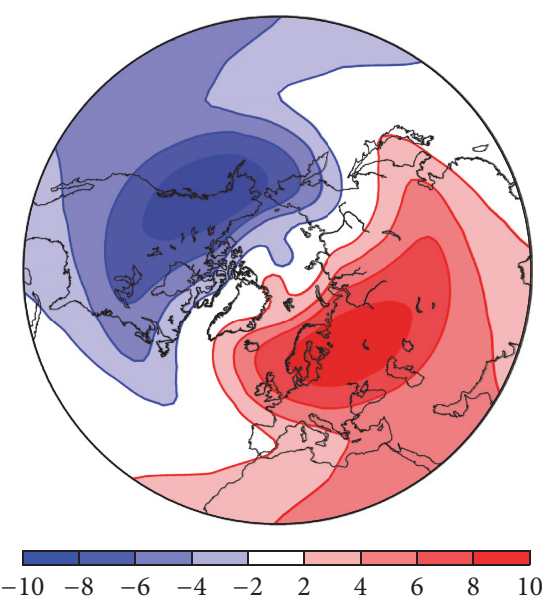

(c)

Figure 5: Zonal wavenumber-1 component of mean geopotential height (gpm) of the $833 \mathrm{hPa}$ model level for the low (a) and high (b) Arctic sea-ice-cover conditions and (c) their difference (low minus high). 


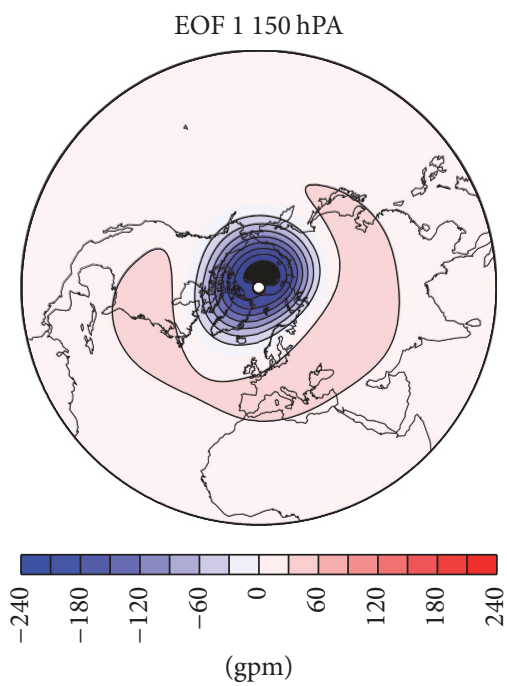

(a)

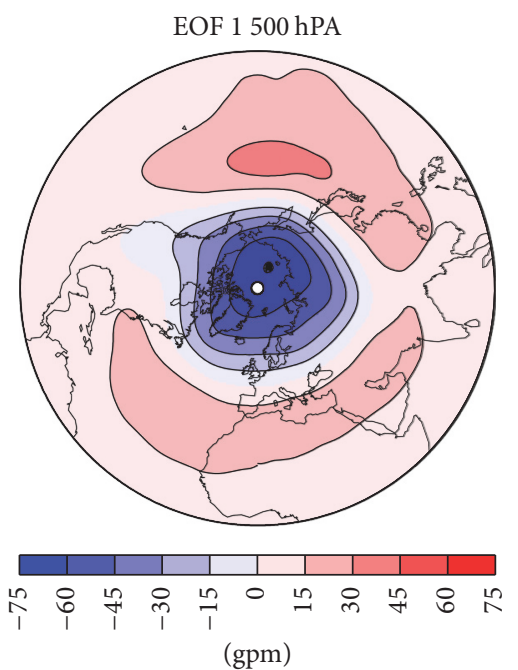

(b)

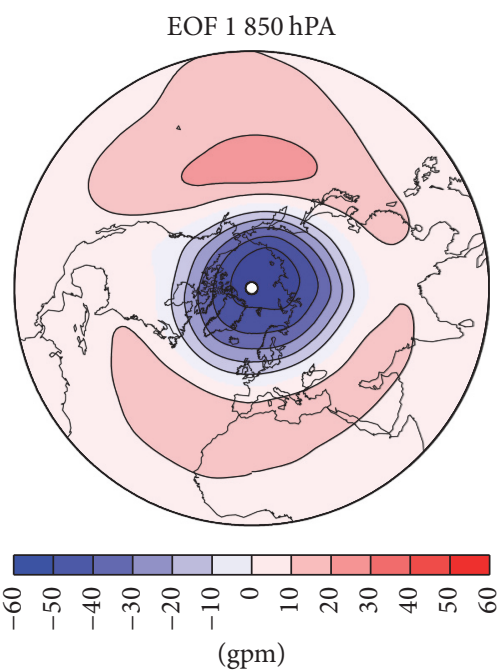

(c)

FIGURE 6: First EOF of geopotential height (gpm) at the three model levels: upper level (a), middle level (b), and lower level (c).

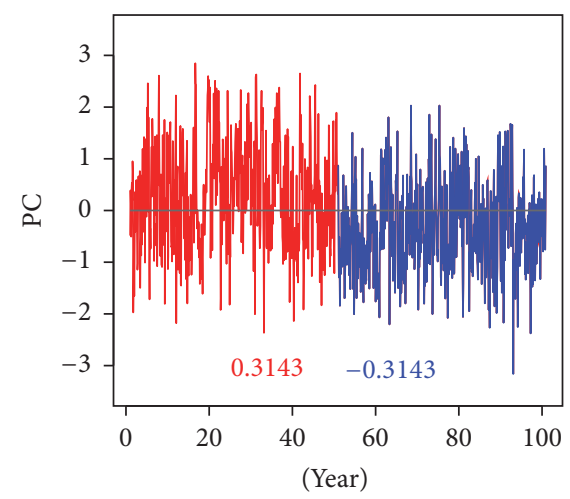

(a)

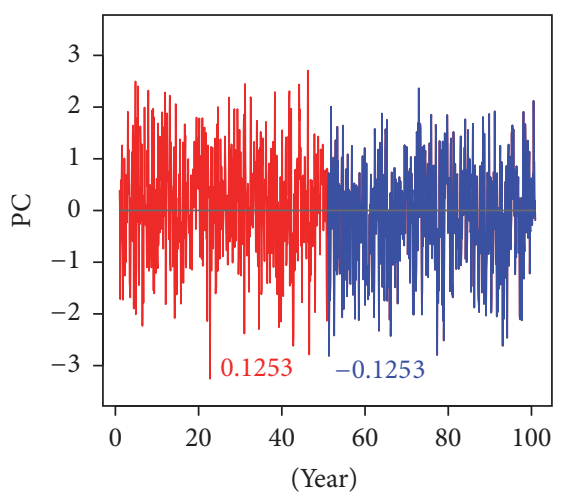

(b)

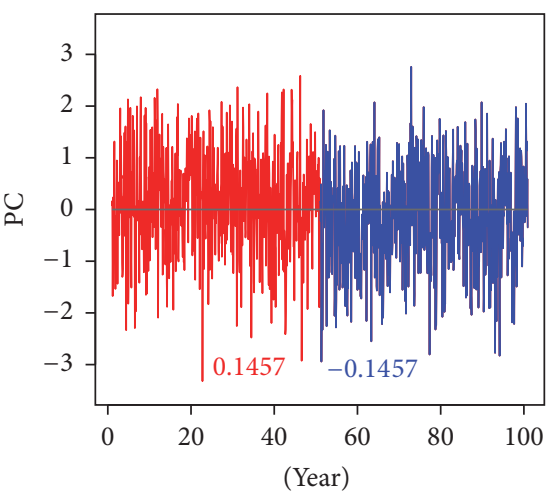

(c)

FIGURE 7: PC time series corresponding to the first EOFs of geopotential height at the three model levels: upper level (a), middle level (b), and lower level (c). The numbers display the mean of the corresponding PC series for the 50-year-long time series. Red and blue colors correspond to the model runs with high and low Arctic sea-ice-cover conditions, respectively.

and its intensity are quite different) with Figure 13 in a model study by Sun et al. [33] applying the Whole Atmosphere Community Climate Model with a well-resolved stratosphere. This figure shows the average over February-March-April of the response of geopotential height (at 1000 and $500 \mathrm{hPa}$ levels) to the Arctic sea-ice concentration loss, which is consistently negative over Pacific. The mechanisms of linkage between $\mathrm{AA}$ and North American wintertime weather anomalies are currently less understood compared to those between AA and anomalies over the North Eurasia, as stated by Overland [31] based on brief literature review on that topic.

Recently, a question has arisen in the literature of the stratospheric versus tropospheric mechanism of linkage between AA and mid-latitude circulation systems (see, [18]). Because of a very coarse vertical resolution (see more in [21]) and enforcedly poor representation of the vertical propagation of planetary-scale waves, it is virtually the tropospheric mechanism of linkage, which acts in the model, and our results show that this mechanism alone is capable of explaining essential features of this linkage. On the other hand, it cannot be excluded that obtained deficiencies (compared to reanalysis) of our modeling results over Pacific and North America are just related to the lack of an efficient and correct stratospheric mechanism of linkage (cf. [33]) and tropical sea surface temperature anomalies in the Pacific.

It is widely accepted that the quasi-geostrophic theory serves as an indispensable tool to understand the physics and dynamics of extratropical atmospheric circulation systems (e.g., [34]). A rather interesting result of this work is that, after proper tuning to compensate for nonadequately resolved processes in the tropical atmosphere and without implementation of explicit ocean-atmosphere interactions and atmospheric moisture processes, our simple dry nonlinear model with its quasi-geostrophic dynamical core is capable 


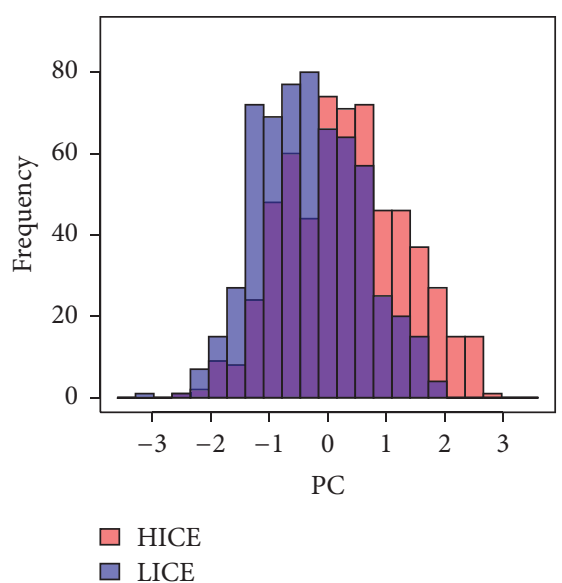

(a)

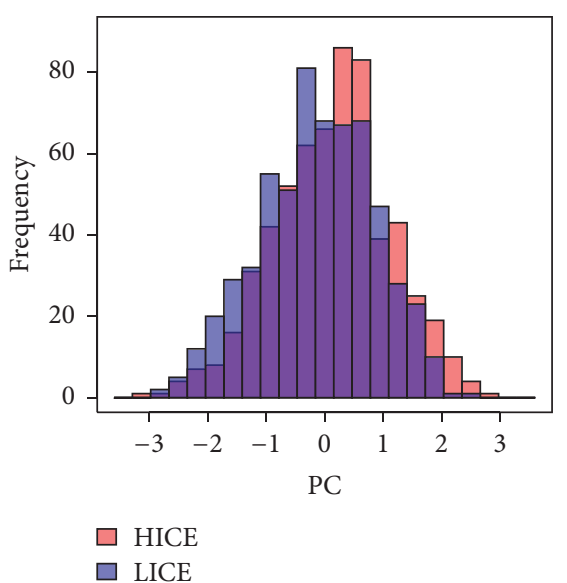

(b)

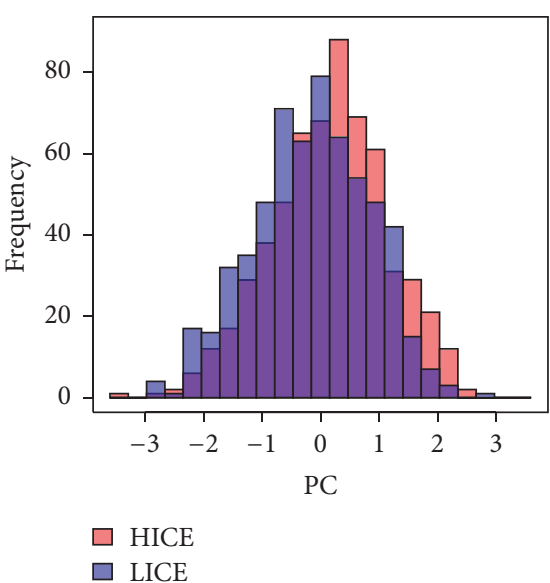

(c)

FIGURE 8: Histograms of corresponding PC time series of geopotential height at the three model levels: upper level (a), middle level (b), and lower level (c). HICE (in red) and LICE (in blue) correspond to the model runs with high and low Arctic sea-ice-cover conditions, respectively. Overlap in the frequency of occurrence between HICE and LICE is marked in purple.

of realistically reproducing the important features of midlatitude atmospheric changes accompanying AA.

Historically, at the very beginning of the era of numerical weather forecasting and atmospheric general circulation modeling, quasi-geostrophic models have played an important role in establishing and putting on firm ground these extremely important directions of atmospheric research. Our results support an idea that the quasi-geostrophic models should not be in complete oblivion nowadays, since they-when properly handled and considered as competent members of a hierarchy of atmospheric models of varying complexity-can help to elucidate principal dynamical mechanisms underlying observed and prospective changes in atmospheric circulation features. In synthesis, our idealized quasi-geostrophic model provides a simplified framework for investigating the dynamical mechanism of Arctic-midlatitude linkage and complements the ongoing efforts with observations and a hierarchy of GCMs.

\section{Data Access}

The output data from the simulations with the quasigeostrophic T63 model can be obtained from the corresponding author (doerthe.handorf@awi.de).

\section{Conflicts of Interest}

The authors declare that there are no conflicts of interest regarding the publication of this paper.

\section{Acknowledgments}

The authors acknowledge the data center of the European Centre for Medium-Range Weather Forecast for providing the ERA-Interim reanalysis (http://apps.ecmwf.int/datasets).

\section{References}

[1] J. C. Stroeve, J. Maslanik, M. C. Serreze, I. Rigor, W. Meier, and C. Fowler, "Sea ice response to an extreme negative phase of the Arctic Oscillation during winter 2009/2010," Geophysical Research Letters, vol. 38, no. 2, Article ID L02502, 2011.

[2] J. A. Screen and I. Simmonds, "The central role of diminishing sea ice in recent Arctic temperature amplification," Nature, vol. 464, no. 7293, pp. 1334-1337, 2010.

[3] M. C. Serreze, A. P. Barrett, J. C. Stroeve, D. N. Kindig, and M. M. Holland, "The emergence of surface-based arctic amplification," Cryosphere, vol. 3, no. 1, pp. 11-19, 2009.

[4] V. A. Alexeev, I. Esau, I. V. Polyakov, S. J. Byam, and S. Sorokina, "Vertical structure of recent arctic warming from observed data and reanalysis products," Climatic Change, vol. 111, no. 2, pp. 215-239, 2012.

[5] J. L. Cohen, J. C. Furtado, M. A. Barlow, V. A. Alexeev, and J. E. Cherry, "Arctic warming, increasing snow cover and widespread boreal winter cooling," Environmental Research Letters, vol. 7, no. 1, Article ID 014007, 2012.

[6] Q. Tang, X. Zhang, X. Yang, and J. A. Francis, "Cold winter extremes in northern continents linked to Arctic sea ice loss," Environmental Research Letters, vol. 8, no. 1, Article ID 014036, 2013.

[7] M. Honda, J. Inoue, and S. Yamane, "Influence of low Arctic seaice minima on anomalously cold Eurasian winters," Geophysical Research Letters, vol. 36, no. 8, Article ID L08707, 2009.

[8] V. Petoukhov and V. A. Semenov, "A link between reduced Barents-Kara sea ice and cold winter extremes over northern continents," Journal of Geophysical Research D: Atmospheres, vol. 115, no. 21, Article ID D21111, 2010.

[9] J. Cohen, J. A. Screen, J. C. Furtado et al., "Recent Arctic amplification and extreme mid-latitude weather," Nature Geoscience, vol. 7, no. 9, pp. 627-637, 2014.

[10] T. Vihma, "Effects of Arctic Sea Ice Decline on Weather and Climate: A Review," Surveys in Geophysics, vol. 35, no. 5, pp. 1175-1214, 2014. 
[11] J. E. Overland, K. Dethloff, J. A. Francis et al., "Nonlinear response of mid-latitude weather to the changing Arctic," Nature Climate Change, vol. 6, no. 11, pp. 992-999, 2016.

[12] R. Jaiser, K. Dethloff, D. Handorf, A. Rinke, and J. Cohen, "Impact of sea ice cover changes on the northern hemisphere atmospheric winter circulation," Tellus, Series A: Dynamic Meteorology and Oceanography, vol. 64, no. 1, Article ID 11595, 2012.

[13] T. Nakamura, K. Yamazaki, K. Iwamoto et al., "A negative phase shift of the winter AO/NAO due to the recent Arctic seaice reduction in late autumn," Journal of Geophysical Research Atmospheres, vol. 120, no. 8, pp. 3209-3227, 2015.

[14] J. Cohen, M. Barlow, P. J. Kushner, and K. Saito, "Stratospheretroposphere coupling and links with Eurasian land surface variability," Journal of Climate, vol. 20, no. 21, pp. 5335-5343, 2007.

[15] M. Wegmann, Y. Orsolini, M. Vázquez et al., "Arctic moisture source for Eurasian snow cover variations in autumn," Environmental Research Letters, vol. 10, no. 5, Article ID 054015, 2015.

[16] R. Jaiser, T. Nakamura, D. Handorf, K. Dethloff, J. Ukita, and K. Yamazaki, "Atmospheric winter response to Arctic sea ice changes in reanalysis data and model simulations," Journal of Geophysical Research: Atmospheres, vol. 121, no. 13, pp. 75647577, 2016.

[17] B.-M. Kim, S.-W. Son, S.-K. Min et al., "Weakening of the stratospheric polar vortex by Arctic sea-ice loss," Nature Communications, vol. 5, 2014.

[18] T. Nakamura, K. Yamazaki, M. Honda et al., "On the atmospheric response experiment to a Blue Arctic Ocean," Geophysical Research Letters, vol. 43, no. 19, pp. 10-402, 2016.

[19] P. Hassanzadeh, Z. Kuang, and B. F. Farrell, "Responses of midlatitude blocks and wave amplitude to changes in the meridional temperature gradient in an idealized dry GCM," Geophysical Research Letters, vol. 41, no. 14, pp. 5223-5232, 2014.

[20] I. M. Held and M. J. Suarez, "A proposal for the intercomparison of the dynamical cores of atmospheric general circulation models," Bulletin of the American Meteorological Society, vol. 75, no. 10, pp. 1825-1830, 1994.

[21] H. Labsch, D. Handorf, K. Dethloff, and M. V. Kurgansky, "Atmospheric circulation regimes in a nonlinear quasigeostrophic model," Advances in Meteorology, vol. 2015, Article ID 629429, 2015.

[22] M. Sempf, K. Dethloff, D. Handorf, and M. V. Kurgansky, "Idealized modelling of the northern annular mode: orographic and thermal impacts," Atmospheric Science Letters, vol. 6, no. 2, pp. 140-144, 2005.

[23] M. Sempf, K. Dethloff, D. Handorf, and M. V. Kurgansky, "Toward understanding the dynamical origin of atmospheric regime behavior in a baroclinic model," Journal of the Atmospheric Sciences, vol. 64, no. 3, pp. 887-904, 2007.

[24] P. L. Houtekamer, "Variation of the predictability in a low-order spectral model of the atmospheric circulation," Tellus, Series A, vol. 43, no. 3, pp. 177-190, 1991.

[25] P. Poli, S. B. Healy, and D. P. Dee, "The ERA-Interim reanalysis: Configuration and performance of the data assimilation system," Quarterly Journal of the Royal Meteorological Society, vol. 136, no. 653, pp. 1972-1990, 2010.

[26] J. C. Adams and P. N. Swarztrauber, "SPHEREPACK 3.0: A model development facility," Monthly Weather Review, vol. 127, no. 8, pp. 1872-1878, 1999.
[27] R. Preisendorfer, Principal Component Analysis in Meteorology and Oceanography, vol. 17, Developments in Atmospheric Science, 1988.

[28] D. Handorf and K. Dethloff, "How well do state-of-the-art atmosphere-ocean general circulation models reproduce atmospheric teleconnection patterns?" Tellus A: Dynamic Meteorology and Oceanography, vol. 64, no. 1, p. 19777, 2012.

[29] H. von Storch and F. W. Zwiers, Statistical Analysis in Climate Research, Cambridge University Press, Cambridge, UK, 1999.

[30] D. Handorf, R. Jaiser, K. Dethloff, A. Rinke, and J. Cohen, "Impacts of Arctic sea ice and continental snow cover changes on atmospheric winter teleconnections," Geophysical Research Letters, vol. 42, no. 7, pp. 2367-2377, 2015.

[31] J. E. Overland, "A difficult Arctic science issue: Midlatitude weather linkages," Polar Science, vol. 10, no. 3, pp. 210-216, 2016.

[32] M. P. Baldwin and T. J. Dunkerton, "Stratospheric harbingers of anomalous weather regimes," Science, vol. 294, no. 5542, pp. 581-584, 2001.

[33] L. Sun, C. Deser, and R. A. Tomas, "Mechanisms of stratospheric and tropospheric circulation response to projected Arctic sea ice loss," Journal of Climate, vol. 28, no. 19, pp. 7824-7845, 2015.

[34] J. R. Holton, An Introduction to Dynamic Meteorology, vol. 88 of International Geophysics, Elsevier, 4th edition, 2004. 

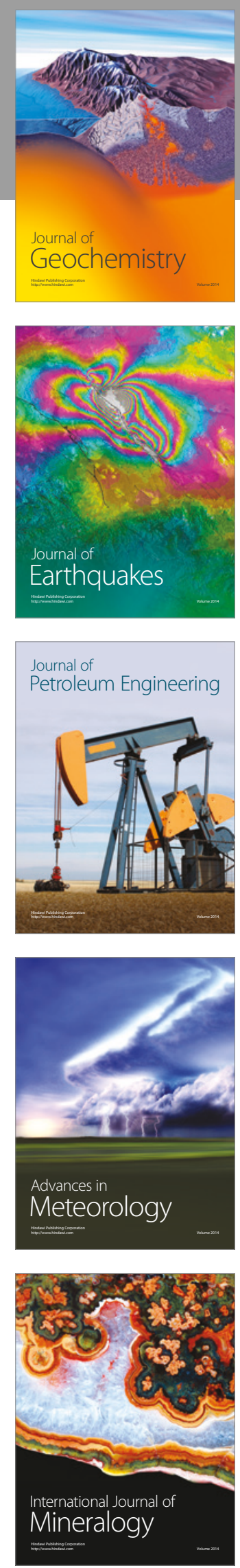
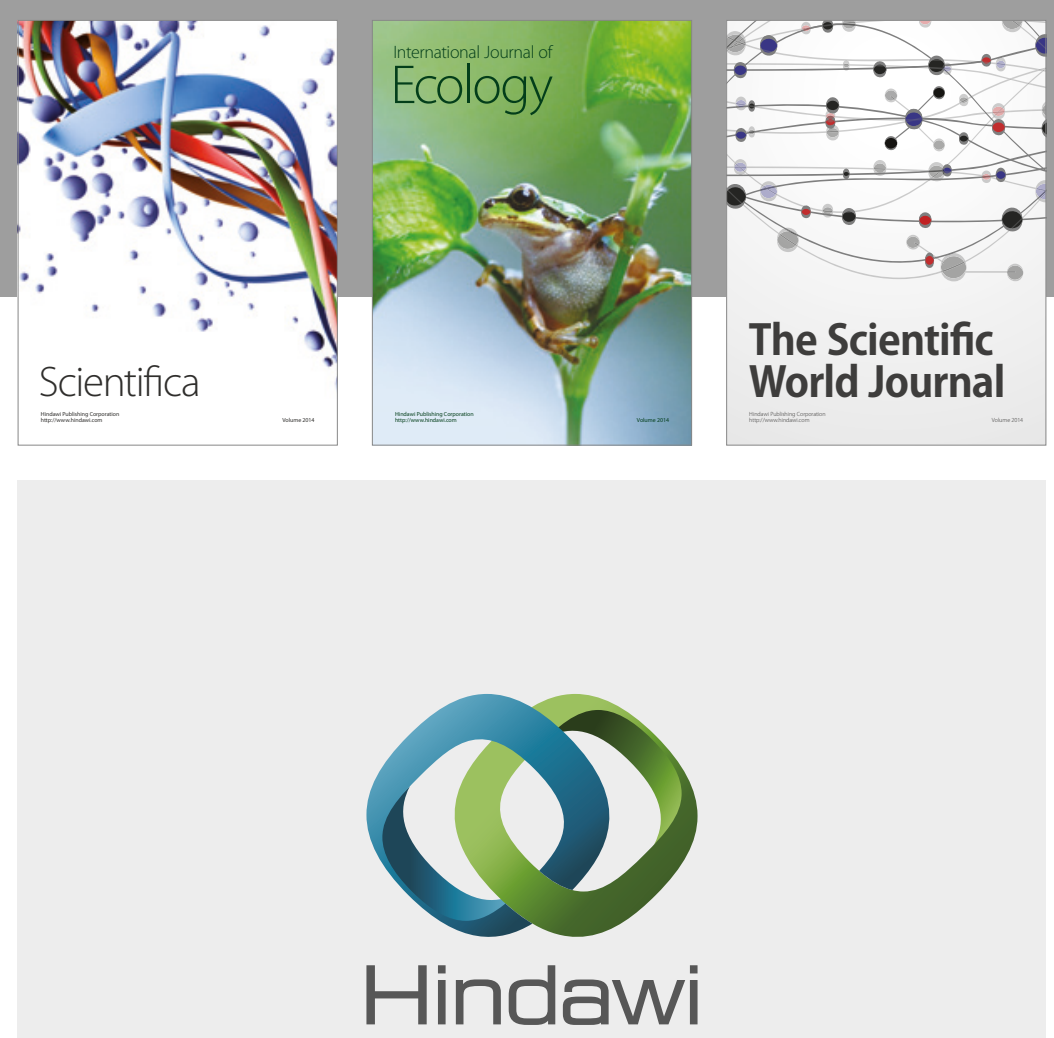

Submit your manuscripts at

https://www.hindawi.com
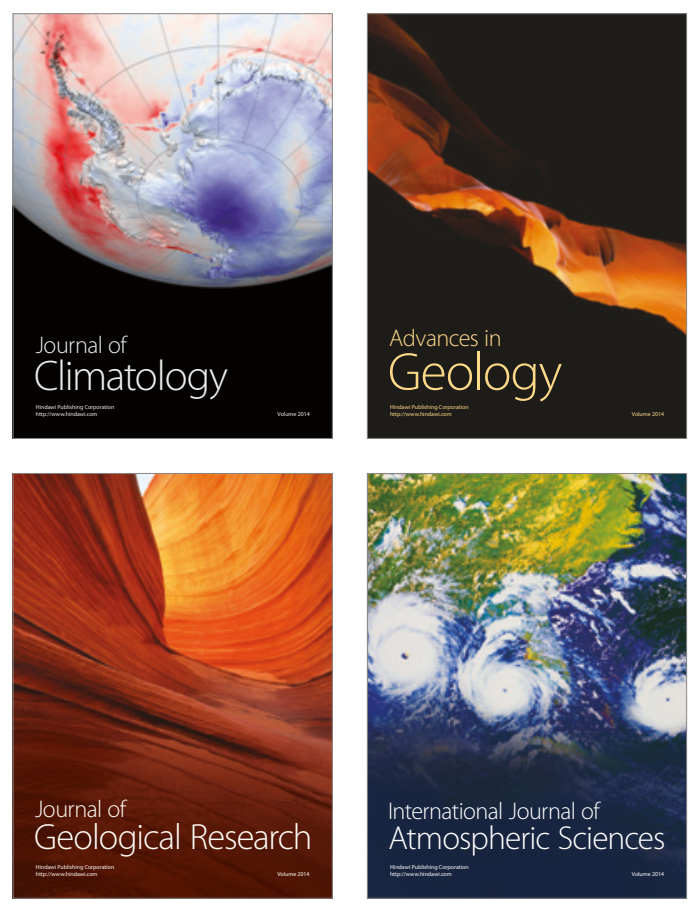

The Scientific

World Journal
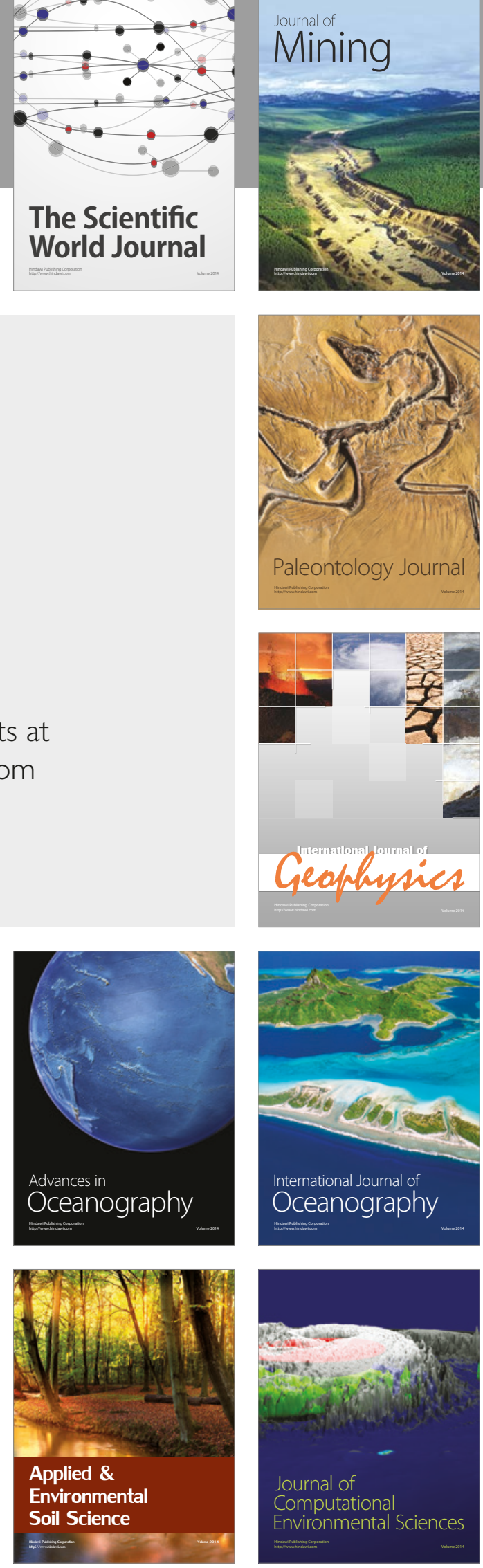